Rev. Int. Contam. Ambie. 35 (Calidad de agua: salud, remediación y perspectiva) 81-91, 2019

DOI: 10.20937/RICA.2019.35.esp03.10

\title{
TRAZABILIDAD DE ARSÉNICO EN AGUA DE RIEGO AGRÍCOLA EN EL CENTRO SUR DEL ESTADO DE CHIHUAHUA, MÉXICO
}

Traceability of arsenic in agricultural irrigation water from center-south of Chihuahua State, Mexico

\author{
Milton Carlos HERMOSILLO-MUÑOZ ${ }^{1}$, María Cecilia VALLES-ARAGÓN ${ }^{1 *}$, \\ María de Lourdes BALLINAS-CASARRUBIAS ${ }^{2}$, Beatriz Adriana ROCHA-GUTIÉRREZ ${ }^{2}$ \\ y Jesús Alejandro PRIETO-AMPARAN ${ }^{3}$
}

\footnotetext{
${ }^{1}$ Facultad de Ciencias Agrotecnológicas, Universidad Autónoma de Chihuahua, Pascual Orozco y Universidad s/n, Campus I. C.P. 31200. Chihuahua, Chih., México

${ }^{2}$ Facultad de Ciencias Químicas, Universidad Autónoma de Chihuahua, Circuito Universitario, Campus II. C.P. 31124. Chihuahua, Chih., México

${ }^{3}$ Facultad de Zootecnia y Ecología, Universidad Autónoma de Chihuahua, Perif. Francisco. R. Almada Km 1, Zootecnia. C.P. 33820. Chihuahua, Chih., México

*Autora para correspondencia: valles.cecilia@gmail.com
}

(Recibido febrero 2108; aceptado octubre 2018)

Palabras clave: contaminación del agua, suelo, cultivos, metaloide

\section{RESUMEN}

La presencia natural de arsénico en agua subterránea es un problema global ya que afecta a la salud humana. El metaloide ha sido detectado en cultivos regados con esta agua, sobre todo en Asia. El objetivo de esta investigación fue determinar la concentración de arsénico total (As-tot) en pozos agrícolas al centro-sur del estado de Chihuahua, así como su trazabilidad a suelo y cultivos del territorio. Se muestrearon 119 pozos de riego agrícola. El muestreo de suelo se realizó en seis sitios, en cada uno se colectaron 24 muestras; en estos mismos se colectaron 32 plantas de cebolla y 37 de chile. La concentración de As-tot se determinó en todas las matrices por espectrometría de absorción atómica con generador de hidruros. El $11 \%$ de los pozos superaron el límite máximo permisible (LMP) de As-tot en agua para uso agrícola $(100 \mu \mathrm{g} / \mathrm{L})$; la mayor concentración de As-tot fue $576 \mu \mathrm{g} / \mathrm{L}$. En el sitio con mayor concentración de As-tot en suelo, el $42 \%$ de las muestras superaron el LMP para uso agrícola ( $22 \mathrm{mg} / \mathrm{kg}$ ), presentando una concentración máxima de $47.5 \mathrm{mg} / \mathrm{kg}$. La mayor presencia de As-tot en cultivos fue en raíz, $7.4 \mathrm{mg} / \mathrm{kg}$ en chile serrano y $28.8 \mathrm{mg} / \mathrm{kg}$ en cebolla. Sin embargo, en fruto se determinó $0.16 \mathrm{mg} / \mathrm{kg}$ en chile y $0.38 \mathrm{mg} / \mathrm{kg}$ en cebolla. Se demostró que en la región el riego de cultivos con agua con altos niveles de As-tot genera movimiento del metaloide a matrices que se relacionan con la ingesta de la población.

Key words: water pollution, soil, crops, metalloid

\begin{abstract}
The natural presence of arsenic in groundwater is a global problem since it affects human health. The metalloid has been detected in crops irrigated with As-contaminated water, especially in Asia. Therefore, the objective of this research was to determine
\end{abstract}


the total As concentration (As-tot) in agricultural wells at the south-central part of Chihuahua State, as well as its traceability to soil and crops in the territory. 119 wells for agricultural irrigation were sampled. The soil sampling was realized in six sites, in each one 24 samples were collected; in the same sites, 32 onion and 37 chili plants were collected. As-tot concentration was determined by atomic absorption spectrometry with hydride generator. $11 \%$ of the wells exceeded the maximum permissible level (MPL) of As-tot in water for agricultural use $(100 \mu \mathrm{g} / \mathrm{L})$. The highest As-tot concentration was $576 \mu \mathrm{g} / \mathrm{L}$. In the quadrant with the highest As-tot concentration in soil, $42 \%$ of the samples exceeded the MPL for agricultural soil $(22 \mathrm{mg} / \mathrm{kg})$, displaying a maximum concentration of $47.5 \mathrm{mg} / \mathrm{kg}$. The highest As-tot presence on plants was in root, 7.4 $\mathrm{mg} / \mathrm{kg}$ in chili and $28.8 \mathrm{mg} / \mathrm{kg}$ in onion. However, $0.16 \mathrm{mg} / \mathrm{kg}$ in chili and $0.38 \mathrm{mg} / \mathrm{kg}$ in onion were also identified in fruits. It was demonstrated that, in the study region, the crops irrigation with water high As levels generates the metalloid movement to matrices that are related to the population intake.

\section{INTRODUCCIÓN}

El arsénico (As) es en un importante tópico de investigación ambiental debido a su toxicidad, abundancia geológica y sus aplicaciones (Quinzhong et al. 2013). Este metaloide es tóxico y su presencia en agua potable causa efectos nocivos y carcinogénicos en los seres humanos (Yousif et al. 2016). Aunado a ello, hay indicios de que el uso de agua contaminada con As para riego agrícola ha dado lugar a la acumulación de As en suelos, lo cual conduce a la bioacumulación de As en plantas comestibles y cosechas (Bundschuh et al. 2012, Shraim 2014, FAO/OMS 2017). El arsenato $\left(\mathrm{As}^{+5}\right)$ y el fosfato $\left(\mathrm{P}^{+5}\right)$ tienen propiedades químicas similares actuando de manera similar en el suelo y plantas. El $\mathrm{P}^{+5}$ y el As ${ }^{+5}$ pueden competir entre sí por los lugares de fijación del suelo y de absorción de las plantas (Punshon et al. 2017). Las hortalizas son los cultivos más expuestos a esta condición, ya que permanecen en contacto directo con el agua y la tierra que los contienen (Ortega García et al. 2010).

En México, muchos municipios, principalmente rurales, cuentan con fuentes de abastecimiento de agua contaminadas con As (Pietro García 2005, Armienta y Segovia 2008, Caballero-Gutiérrez 2010, Vega-Gleason 2013). En el estado de Chihuahua destacan los municipios de Jiménez, Camargo, Delicias, Meoqui, Julimes y Rosales, entre otros, en los cuales se han reportado concentraciones de As mayores a las permitidas por la normatividad para agua de consumo humano (Olmos-Márquez 2011). Espino-Valdés et al. (2009) determinaron en pozos de consumo humano que el $72 \%$ de las 61 muestras analizadas excedieron el límite máximo permisible (LMP) de As total (As-tot) para agua de consumo humano establecido por la normativa mexicana
NOM-127-SSA1-1994 de $25 \mu \mathrm{g} / \mathrm{L}$ (SSA 1994) y el $18 \%$ superó los $100 \mu \mathrm{g} / \mathrm{L}$, al norte del acuífero Meoqui-Delicias (ubicado al centro-sur del estado de Chihuahua). Olmos-Márquez (2011) determinó en 12 municipios del estado de Chihuahua que el LMP de As para agua de consumo humano en la alimentación de 116 plantas de osmosis inversa se excedió en un $73 \%$, y en $5 \%$ de los pozos muestreados excedió los $100 \mu \mathrm{g} / \mathrm{L}$. Por otro lado, Arreguín-Cortés et al. (2013) definieron que las concentraciones de 139 muestras de agua de consumo humano en la región centro-sur del estado, se encontraron en un intervalo de $<2$ a $225 \mu \mathrm{g} / \mathrm{L}$ de As-tot. Asimismo, González-Horta et al. (2015) en un estudio en la región suroeste del estado de Chihuahua, reportaron altos niveles de As-tot en agua de consumo humano y el $49 \%$ de personas muestreadas presentaron altos niveles de As-tot en orina. Dichas investigaciones evidencian la presencia del metaloide en la región, lo que puede representar un factor para su incorporación en otras matrices del medio físico que intervenga en las actividades económicas y pongan en riesgo la salud de la población.

En el centro sur del estado de Chihuahua la actividad económica más importante es la agricultura, donde se siembra avena forrajera, trigo forrajero, algodón, cacahuate, cebolla, chile, maíz forrajero, sandía, melón, alfalfa y nogal, entre otros (INEGI 2013). Uno de los principales suministros de agua en dicho territorio para esta actividad es el agua subterránea proveniente del acuífero Meoqui-Delicias, el cual cuenta con cerca de 1000 aprovechamientos y se encuentra en condición de sobreexplotación debido a la sequía padecida en el estado de Chihuahua años atrás. Este fenómeno, además de impactar negativamente en el volumen de agua disponible, afectó también la calidad de los recursos hidrológicos (Espino Valdés et al. 2009). 
Asimismo, el As presente en el agua de riego y el suelo agrícola puede acceder a las plantas a través de sus sistemas de raíces, lo que resulta en efectos fitotóxicos para algunos cultivos (Rosas-Castor et al. 2014).

Por lo anterior, se consideró que además del agua de consumo humano, que ya está demostrado contiene altas concentraciones de As-tot, los cultivos regados con agua contaminada con As podrían resultar un aporte a la ingesta diaria de As en la alimentación de la población si dichas plantas traslocan el metaloide a la parte comestible (Dahal et al. 2008, Chandra Santra et al. 2013).

Con base en estos hallazgos y en que la calidad del agua y suelo de uso agrícola resulta relevante en la producción e inocuidad de alimentos (CaballeroGutiérrez 2010, Suresh 2015), el objetivo de esta investigación fue determinar la concentración de As-tot en pozos de riego agrícola de la región centro sur del estado de Chihuahua, así como la trazabilidad de este metaloide en suelo y plantas para consumo humano.

\section{MATERIALES Y MÉTODOS}

\section{Área de estudio}

La presente investigación se realizó en la región centro sur del estado de Chihuahua, en el Distrito de
Riego 005 (DR-005) administrado por la Comisión Nacional del Agua (CONAGUA). Se localiza en las coordenadas geográficas $105^{\circ} 40^{\prime} \mathrm{O} 28^{\circ} 30^{\prime} \mathrm{N}$, $105^{\circ} 20^{\prime}$ O $28^{\circ} 30^{\prime} \mathrm{N}, 105^{\circ} 40^{\prime} \mathrm{O} 28^{\circ} 10^{\prime} \mathrm{N}, 105^{\circ} 20^{\prime}$ O $28^{\circ} 10^{\prime} \mathrm{N}$ y altitud de $1156 \mathrm{msnm}$. El DR-005 está compuesto por 10 módulos de riego, que son administrados por asociaciones locales, los cuales se ubican sobre el área territorial de los municipios de Julimes, Rosales, Delicias, Meoqui, La Cruz, Saucillo y Camargo (Fig. 1).

\section{Muestreo de agua, suelo y plantas}

Se realizaron dos muestreos de agua de los pozos de la zona de estudio, administrados por el DR-005 y en funcionamiento, el primero en verano (muestreo 1) y el segundo realizado en otoño (muestreo 2 ) del mismo año 2015 (Fig. 2), siendo algunos de los pozos diferentes por temporada. Se siguió el procedimiento establecido en la norma NOM-014-SSA1-1993 (SSA 1993), se tomaron muestras de $1 \mathrm{~L}$, a las cuales se les adicionaron $2 \mathrm{~mL}$ de ácido nítrico $\left(\mathrm{HNO}_{3}\right)$ para su preservación y se mantuvieron en refrigeración desde el momento de la toma. En verano (muestreo 1) se colectaron muestras de 65 pozos, en otoño (muestreo 2) se colectaron muestras de 54 pozos.

Para el muestreo de suelo en el verano se consideraron las zonas que excedieron el LMP $(100 \mu \mathrm{g} / \mathrm{L})$
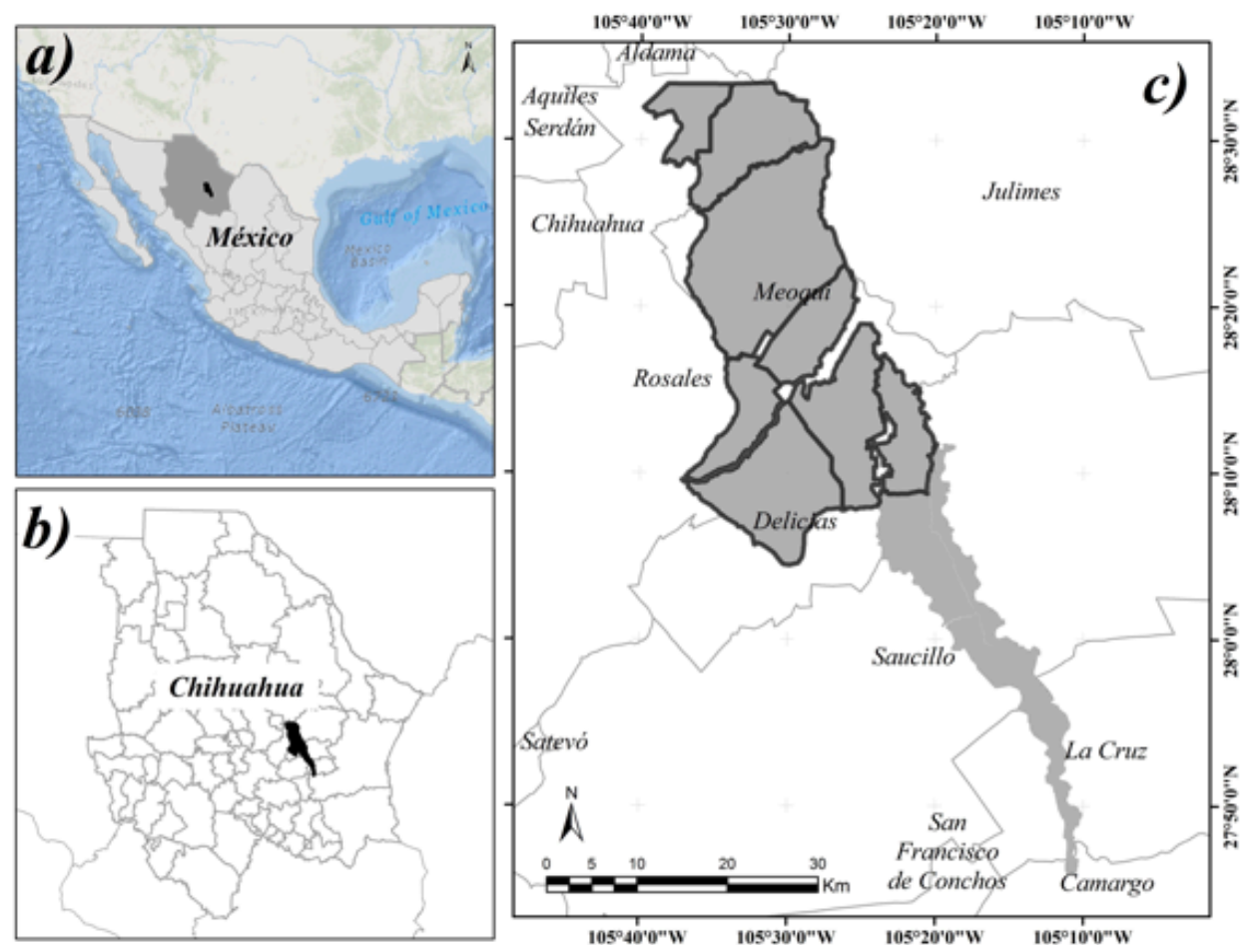

Fig. 1. Área del Distrito de Riego 005. a) Localización del distrito de riego en México, b) Localización del distrito de riego en Chihuahua c) a Distrito de Riego 005. (-) Límite del área de estudio 
a)

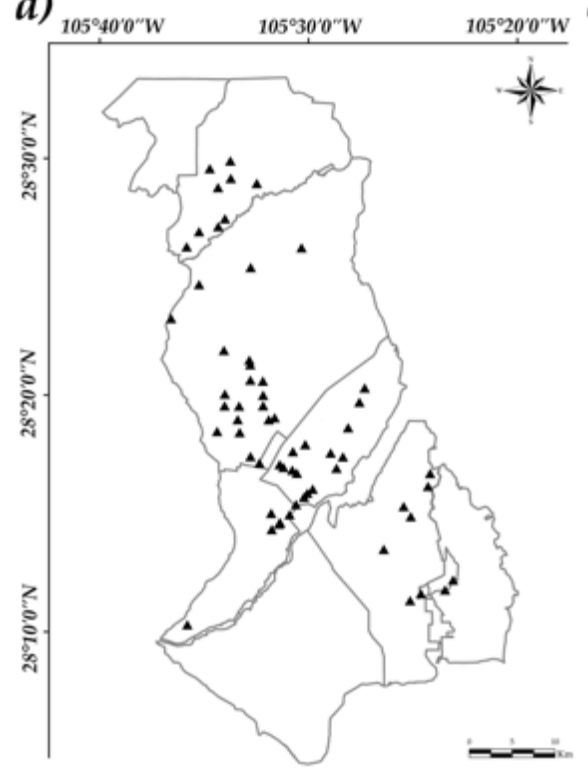

b)

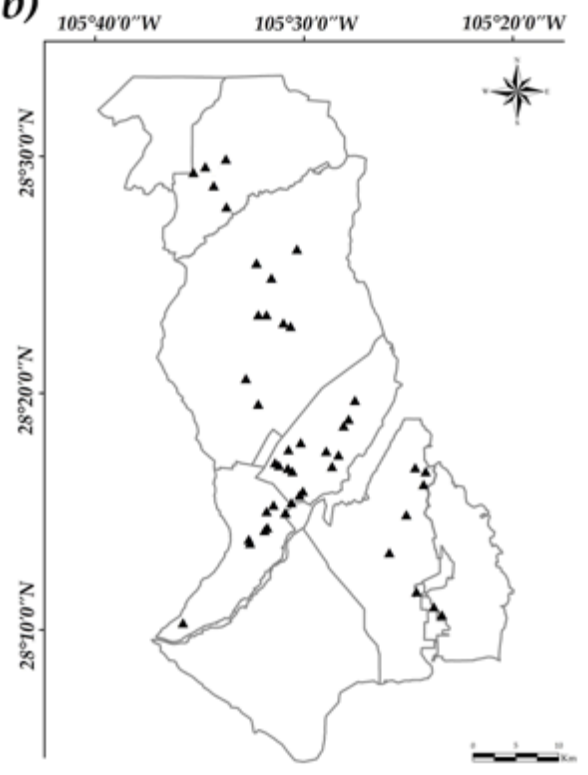

Fig. 2. Muestreo de agua: a) verano, muestreo 1 y b) otoño, muestreo 2. Módulos en estudio $(-)$. Sitios de muestreo $(\mathbf{\Delta})$

de acuerdo con la normativa mexicana para el contenido de As en agua de uso agrícola de (CONAGUA 2016). Se identificaron seis sitios localizados en el municipio de Meoqui, donde se realizó un muestreo sistemático por cuadrantes en cada uno de ellas, según lo establecido en la NMX-AA132-SCFI-2006 (SCFI 2006). Se definieron seis cuadrantes conformados por 24 puntos de muestreo a una distancia de $250 \mathrm{~m}$ entre ellos, en un área de $100 \mathrm{Ha}$ alrededor del pozo de agua, como se observa en la figura 3. Sólo en uno de los
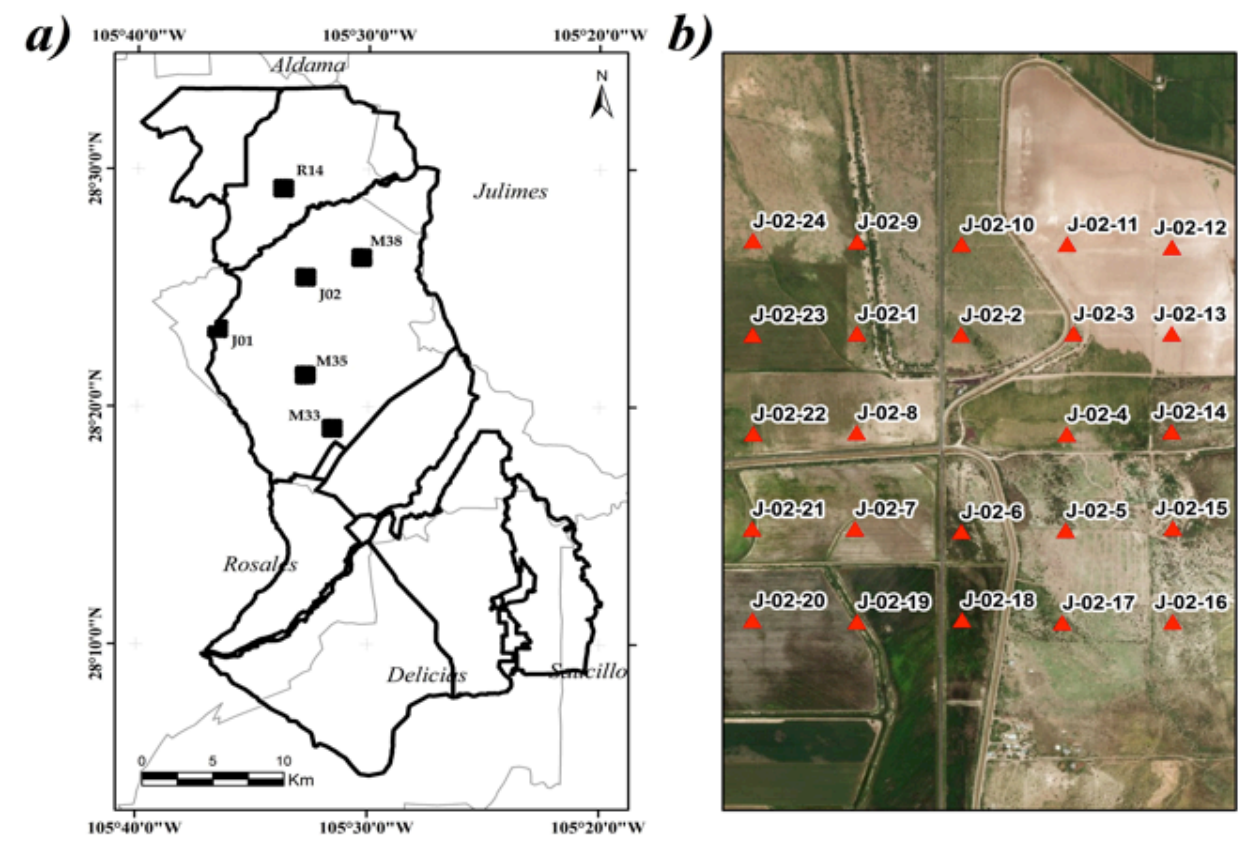

Fig. 3. Muestreo de suelo. a) ejemplificación de los cuadrantes conformados, b) puntos de muestreo. Módulos en estudio (-), cuadrantes ( $\mathbf{\square})$, sitios de muestreo $(\boldsymbol{\Delta})$ 
cuadrantes por su cercanía a una zona habitacional se omitió la colecta de cuatro muestras. Los cuadrantes se denominaron: J01, J02, M33, M35, M38 y R14, recibiendo la nomenclatura que ya tenía el pozo analizado previamente en la zona. En total, se colectaron 140 muestras de suelo y fueron secadas en horno a $35^{\circ} \mathrm{C}$, como se indica en la NMX-AA132-SCFI-2006 (SCFI 2006).

Para el muestreo de plantas se identificaron los cultivos regados en los cuadrantes de estudio previamente definidos en el párrafo anterior. Se priorizó la identificación de cultivos como chile y cebolla debido a su alto consumo en México. Para la recolección de las muestras se recorrieron las parcelas en temporada de cosecha de cultivos que se riegan con agua subterránea (de mayo a julio de 2016). Se recolectaron 32 muestras de planta de cebolla, 23 de chile jalapeño, cuatro de chile serrano y diez de chile chilaca. Las muestras se enjuagaron con agua destilada para retirar el suelo y secaron a $45^{\circ} \mathrm{C}$ (Litter 2009) y luego se seccionaron en raíz, tallo, hoja y fruto. Cada parte de la planta se molió y almacenó en bolsas de plástico.

\section{Preparación y análisis de las muestras}

Las muestras de agua se filtraron con papel Whatman No. 41 libre de cenizas de $0.2 \mathrm{~mm}$ de poro; posteriormente antes del análisis se filtraron con membranas Milipore de $0.45 \mu \mathrm{m}$ de poro. Para la digestión de las muestras de suelo y plantas se utilizó un microondas CEM Mars 6. Para el suelo se utilizó el método EPA-3052, empleando $0.5 \mathrm{~g}$ de muestra, $9 \mathrm{~mL}$ de $\mathrm{HNO}_{3}$ concentrado y $3 \mathrm{~mL}$ de ácido hidrofluorídrico. Las plantas se digirieron de acuerdo con el método EPA-3051, utilizando $0.25 \mathrm{~g}$ de muestra y $9 \mathrm{~mL}$ de $\mathrm{HNO}_{3}$, dejando las muestras en predigestión $12 \mathrm{~h}$.

La determinación de As-tot en todas las matrices se realizó con un equipo de espectrometría de absorción atómica Perkin Elmer AAnalyst 700, al cual se acopló un generador de hidruros FIAS 100, siguiendo la norma NMX-AA-051-SCFI-2001 (SCFI 2001). El límite de detección del equipo fue de $3.12 \mu \mathrm{g} / \mathrm{L}$, las muestras se analizaron por triplicado, utilizando el estándar Trace Metals-Sand 1 Número CRM048 de Sigma Aldrich con un porcentaje de recuperación del $99 \%$.

\section{Distribución espacial de arsénico y análisis esta- dístico}

Para analizar la distribución espacial del As-tot en agua y en suelo se utilizó el método de distancia inversa ponderada (IDW, por sus siglas en inglés) del programa ArcMap $10.3^{\circ}$. El método de IDW para estimar la concentración de los lugares no muestreados utiliza las concentraciones existentes que se encuentran alrededor del área a estimar. Las concentraciones de las observaciones más cercanas tendrán mayor influencia que las que están más lejos, es decir, la influencia disminuye con la distancia (Moreno 2008).

El análisis estadístico de los datos se realizó con el programa Minitab 17, se determinó la correlación de As-tot entre temporadas y se definieron estadísticos descriptivos básicos (media, desviación estándar, intervalo).

\section{RESULTADOS Y DISCUSIÓN}

\section{Arsénico en agua}

En el muestreo 1 (verano) $9 \%$ de los pozos sobrepasaron el LMP de As-tot para riego agrícola y $38 \%$ el LMP para consumo humano de $25 \mu \mathrm{g} / \mathrm{L}$ (SSA 1994); la máxima concentración fue de 335 $\mu \mathrm{g} / \mathrm{L}$. En el muestreo 2 (otoño) se determinó que $13 \%$ de los pozos sobrepasaron el LMP para riego agrícola y $33 \%$ el LMP para consumo humano. La máxima concentración de As-tot presente fue $576 \mu \mathrm{g} / \mathrm{L}$ (Cuadro I).

CUADRO I. ARSÉNICO TOTAL EN MUESTRAS DE AGUA DEL CENTRO SUR DEL ESTADO DE CHIHUAHUA

\begin{tabular}{|c|c|c|c|c|c|c|c|c|}
\hline \multicolumn{2}{|c|}{ Muestras } & \multicolumn{2}{|c|}{ LMP riego agrícola } & \multicolumn{2}{|c|}{ LMP consumo humano } & \multicolumn{2}{|c|}{ No detectables } & Intervalo \\
\hline \multirow[b]{2}{*}{ Muestreo } & \multirow{2}{*}{$\begin{array}{l}\text { Tamaño } \\
\text { muestral } \\
\text { (n) }\end{array}$} & \multicolumn{2}{|c|}{$>100 \mu \mathrm{g} / \mathrm{L}$} & \multicolumn{2}{|c|}{$>25 \mu \mathrm{g} / \mathrm{L}$} & \multicolumn{2}{|c|}{$<3 \mu \mathrm{g} / \mathrm{L}$} & \multirow[b]{2}{*}{$\mu \mathrm{g} / \mathrm{L}$} \\
\hline & & $\begin{array}{l}\text { Porcentaje } \\
\quad(\%)\end{array}$ & $\begin{array}{c}\text { Tamaño } \\
\text { muestral (n) }\end{array}$ & $\begin{array}{l}\text { Porcentaje } \\
(\%)\end{array}$ & $\begin{array}{c}\text { Tamaño } \\
\text { muestral (n) }\end{array}$ & $\begin{array}{l}\text { Porcentaje } \\
(\%)\end{array}$ & $\begin{array}{c}\text { Tamaño } \\
\text { muestral (n) }\end{array}$ & \\
\hline Global & 119 & 11 & 13 & 36 & 43 & 43 & 51 & $<3-576$ \\
\hline 1 & 65 & 9 & 6 & 38 & 25 & 45 & 29 & $<3-335$ \\
\hline 2 & 54 & 13 & 7 & 33 & 18 & 41 & 22 & $<3-576$ \\
\hline
\end{tabular}

LMP Riego Agrícola (CONAGUA 2016), LMP Consumo Humano (SSA 1994) 

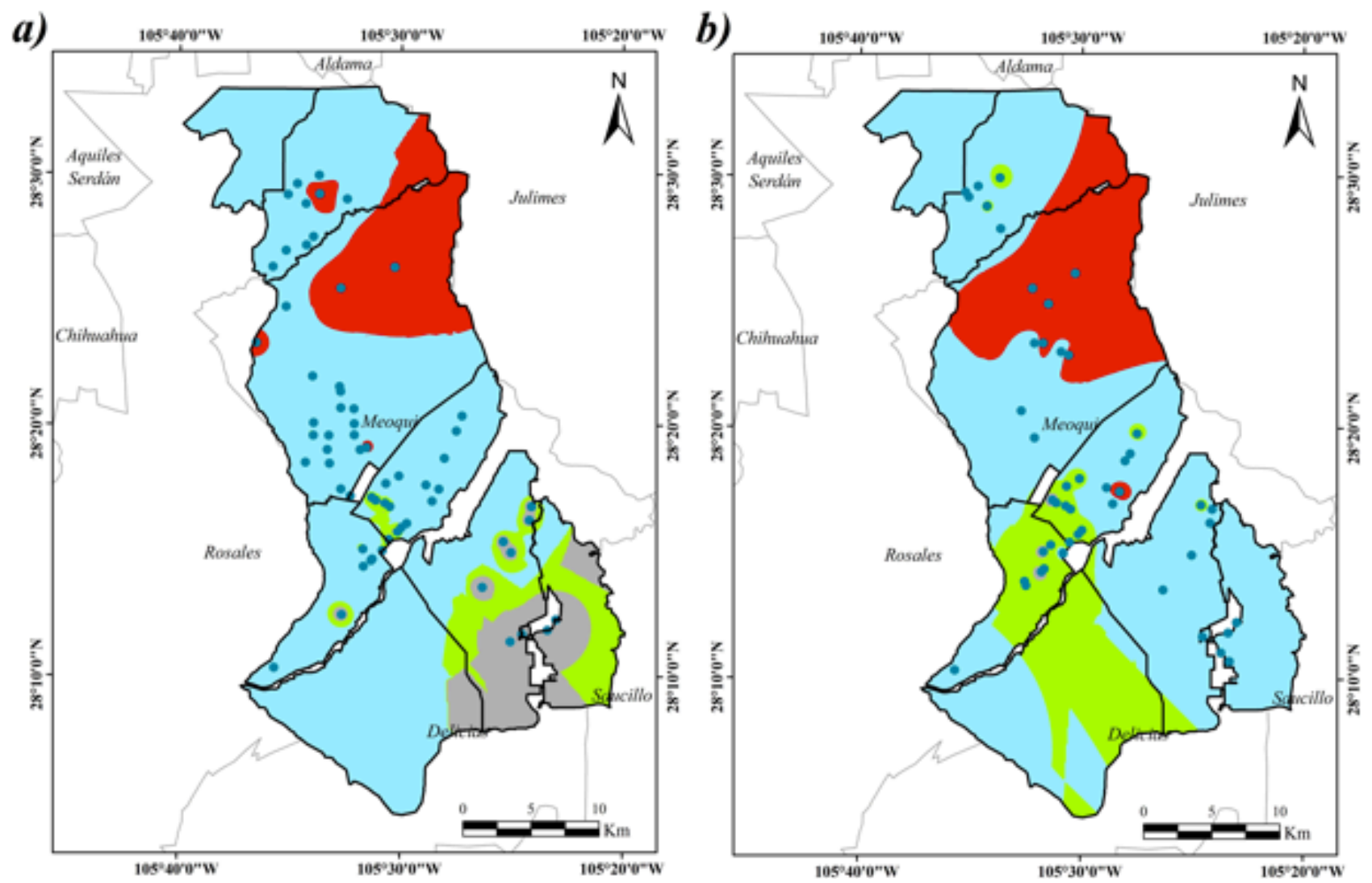

Fig. 4. Dispersión espacial del arsénico total ( $\mu \mathrm{g} / \mathrm{L})$ en agua. a) verano, muestreo 1 y b) otoño, muestreo 2 . (ロ) 0 a 10 , (घ) 10 a 25 , (ロ)

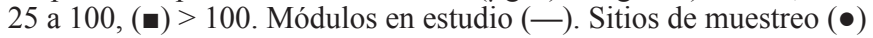

En la figura 4 se muestra la dispersión geográfica de As-tot en agua en la zona de estudio, donde se compararon las dos temporadas muestreadas. Las máximas concentraciones de As-tot se encontraron en la parte norte del DR-005 (color rojo). Asimismo, se observó una extensión territorial predominante que superó el LMP para consumo humano (rojo y azul). No hubo variabilidad entre los pozo que se muestrearon en ambas temporadas, pues al compararlos se obtuvo un $\mathrm{R}^{2}=0.86$

Las concentraciones de As-tot en agua de pozos de riego agrícola del DR-005 $(<3$ a $576 \mu \mathrm{g} / \mathrm{L})$, representaron la problemática de la presencia del metaloide en la región a niveles semejantes o mayores a los reportados por otros autores en pozos para consumo humano en la misma zona: $<25$ a $376 \mu \mathrm{g} / \mathrm{L}$ (Espino-Valdés et al. 2009), $<1$ a 157.38 $\mu \mathrm{g} / \mathrm{L}$ (Olmos-Márquez 2011), < 2 a $225 \mu \mathrm{g} / \mathrm{L}$ (Arreguín-Cortés et al. 2013). Estas concentraciones son semejantes o mayores a las reportadas en otras regiones agrícolas del país como Zimapán, que presenta un problema de contaminación de As en agua generado por actividades mineras, donde se reportó un rango de concentración de As en agua de riego de 40-480 $\mu \mathrm{g} / \mathrm{L}$ (Prieto-García et al., 2005). En Flor de Guayabal, Oaxaca, que presenta problemas de contaminación de As de fuentes naturales, fueron reportadas concentraciones de As-tot en agua en un rango de 43-192 $\mu \mathrm{g} / \mathrm{L}$. Finalmente, en la cuenca de Pilcomayo, Bolivia, en una zona agrícola con actividades mineras, se reportaron concentraciones de As-tot en agua de 50 a $200 \mu \mathrm{g} / \mathrm{L}$ (Bundschuh et al. 2012).

\section{Arsénico en suelo}

En la figura 5 se observa la modelación de la dispersión de As-tot en las áreas de muestreo de suelo en cada cuadrante (niveles arriba del LMP en rojo). El sitio con mayores concentraciones de As-tot fue el J02 con $42 \%$ de un total de 24 muestras que superaron el LMP establecido de $22 \mathrm{mg} / \mathrm{kg}$ (SEMARNAT/SSA 2004) para Astot en suelo residencial o agrícola y un rango de concentración de 8.7 a $47.5 \mathrm{mg} / \mathrm{kg}$ (Cuadro II). Los sitios en que todas las muestras estuvieron dentro 
a)

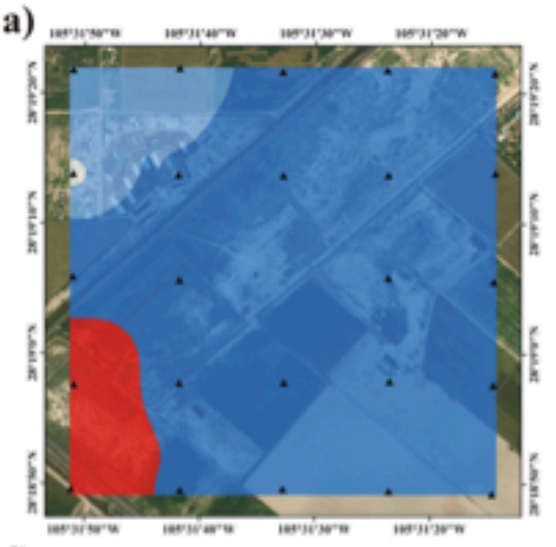

d)

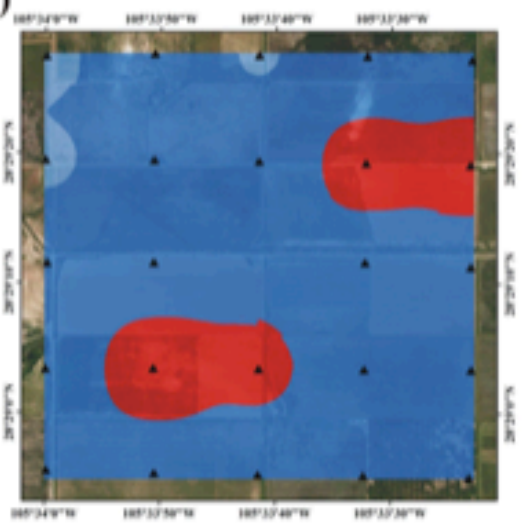

b)

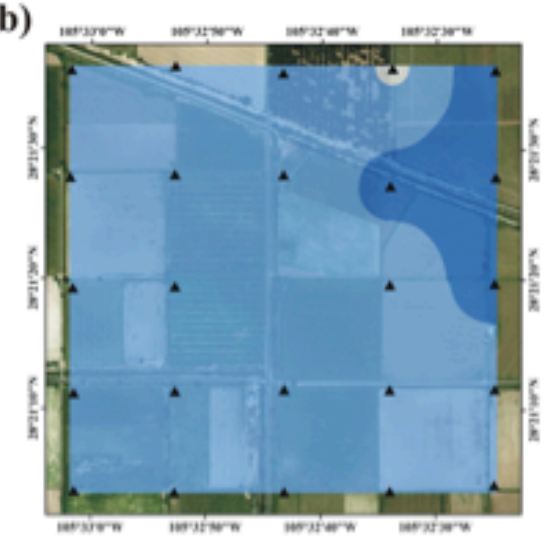

e)

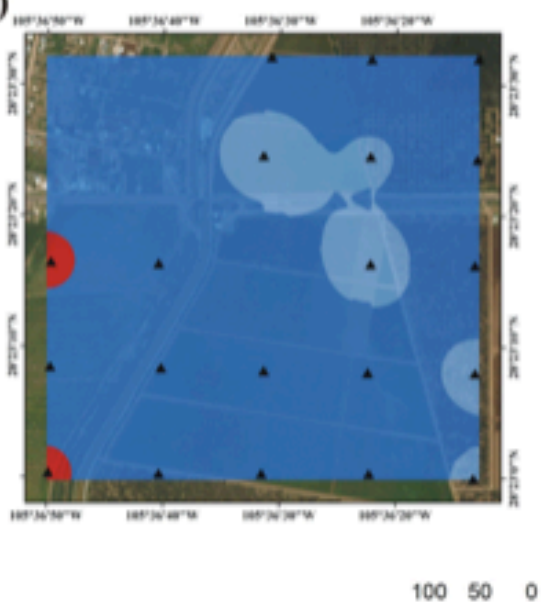

c)

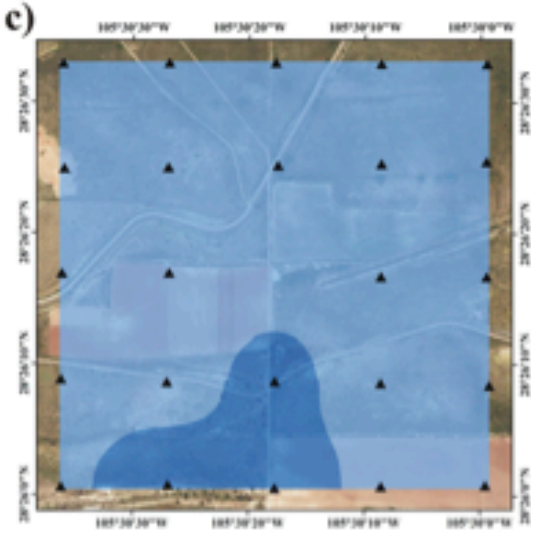

f)
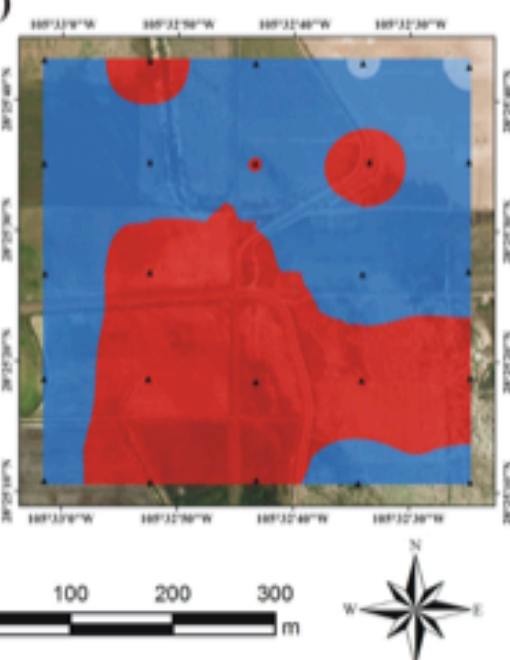

Fig. 5. Dispersión del arsénico total ( $\mathrm{mg} / \mathrm{kg}$ ) en suelo en los seis cuadrantes muestreados. a) M33, b) M35, c) M38, d) R14, e) J01, f)

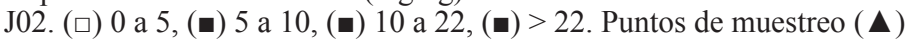

del LMP fueron M35 y M38, presentando rangos de concentración de As de 4.3 a 15.6 y 5.8 a $13.5 \mathrm{mg} /$ $\mathrm{kg}$, respectivamente.
Aunque no todos los sitios presentaron concentraciones elevadas de As-tot en suelo (Fig. 5), se evidenció la presencia de As-tot en todas las muestras de

CUADRO II. ARSÉNICO TOTAL EN MUESTRAS DE SUELO EN EL CENTRO SUR DEL ESTADO DE CHIHUAHUA

\begin{tabular}{|c|c|c|c|c|}
\hline \multirow{2}{*}{ Nomenclatura } & \multirow{2}{*}{$\begin{array}{c}\text { Tamaño } \\
\text { muestral (n) }\end{array}$} & \multicolumn{2}{|c|}{$\begin{array}{l}\text { LMP Residencial/Agrícola } \\
\quad>22 \mathrm{mg} / \mathrm{kg}\end{array}$} & \multirow{2}{*}{$\begin{array}{c}\text { Intervalo } \\
\mathrm{mg} / \mathrm{kg}\end{array}$} \\
\hline & & Porcentaje $(\%)$ & Tamaño muestral (n) & \\
\hline Global & 140 & 13 & 18 & $4.3-57.7$ \\
\hline M33 & 24 & 8 & 2 & $4.4-57.7$ \\
\hline M35 & 24 & 0 & 0 & $4.3-15.6$ \\
\hline M38 & 24 & 0 & 0 & $5.8-13.5$ \\
\hline R14 & 24 & 17 & 4 & $7.9-35.6$ \\
\hline J01 & 20 & 10 & 2 & $8.3-23.5$ \\
\hline $\mathrm{J} 02$ & 24 & 42 & 10 & $8.7-47.5$ \\
\hline
\end{tabular}

LMP Residencial/Agrícola (SEMARNAT/SSA 2004). 
suelo analizadas, en las que el rango de concentración global del metaloide fue de 4.3 a $57.7 \mathrm{mg} / \mathrm{kg}$. En Zimapán, el rango de concentración de As-tot reportado fue mucho menor $(0.72$ a $21 \mathrm{mg} / \mathrm{kg})$ que el obtenido en esta investigación (Prieto-García et al. 2005). En la cuenca de Pilcomayo, Bolivia, la mayoría de los suelos presentaron concentraciones de As-tot mayores en un rango de 30 a $60 \mathrm{mg} / \mathrm{kg}$ (Bundschuh et al. 2012). Por otra parte, la concentración de As-tot promedio que se considera para el suelo a nivel global es de $6.83 \mathrm{mg} / \mathrm{kg}$ (Kabata-Pendias 2011).

Sin embargo, la concentración de As-tot en suelo no define la fitodisponibilidad de este elemento. Debido a que una cantidad finita de As-tot en el suelo es fácilmente móvil, el resto no está disponible para las plantas debido a que este metaloide se asocia con hierro $(\mathrm{Fe})$ y aluminio ( $\mathrm{Al})$, y asimismo las propiedades del suelo también son importantes en la determinación de su disponibilidad (Kabata-Pendias 2011).

\section{Arsénico en plantas}

El análisis de plantas de chile jalapeño, serrano y chilaca mostró mayor concentración de As-tot en la raíz; los valores medios fueron $0.32,7.4$ y $1.2 \mathrm{mg} /$ $\mathrm{kg}$, respectivamente. En las hojas la concentración media fue de $0.53,0.7$ y $1.2 \mathrm{mg} / \mathrm{kg}$, respectivamente. Aunque, la parte comestible o fruto del chile presentó los niveles más bajos de As-tot, éste se acumuló con un valor medio de $0.16 \mathrm{mg} / \mathrm{kg}$ en jalapeño, $0.05 \mathrm{mg} /$ $\mathrm{kg}$ en serrano y no se detectó presencia del metaloide en chilaca (Fig. 6). En Zimapán la concentración de

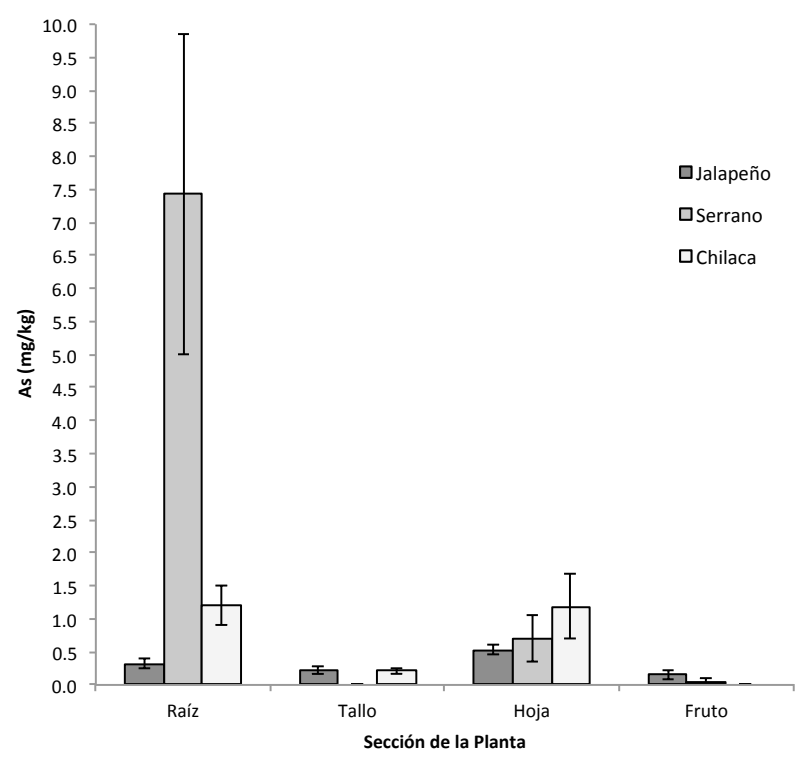

Fig. 6. Arsénico total ( $\mathrm{mg} / \mathrm{kg})$ en las plantas de chile jalapeño, serrano y chilaca
As-tot reportada en el fruto de chile fue mucho mayor que en esta investigación $(6.3 \mathrm{mg} / \mathrm{kg}$ ) (Prieto-García et al. 2005).

El análisis de las plantas de cebolla mostró la mayor concentración de As-tot en la raíz, con un valor promedio de $28.8 \mathrm{mg} / \mathrm{kg}$. Además, se observó que éste llega a acumularse en el bulbo de la cebolla en una cantidad mayor que en el fruto chile. Se determinó una concentración media de $0.38 \mathrm{mg} / \mathrm{kg}$ de As-tot en el bulbo (Fig. 7). Este valor es cercano a los niveles más bajos $(0.35 \mathrm{mg} / \mathrm{kg})$ presentados en el bulbo de la cebolla en Pilcomayo, Bolivia (Bundschuh et al. 2012) y es menor al reportado para Zimapán $(0.7 \mathrm{mg} / \mathrm{kg})$ (Prieto-García et al. 2005).

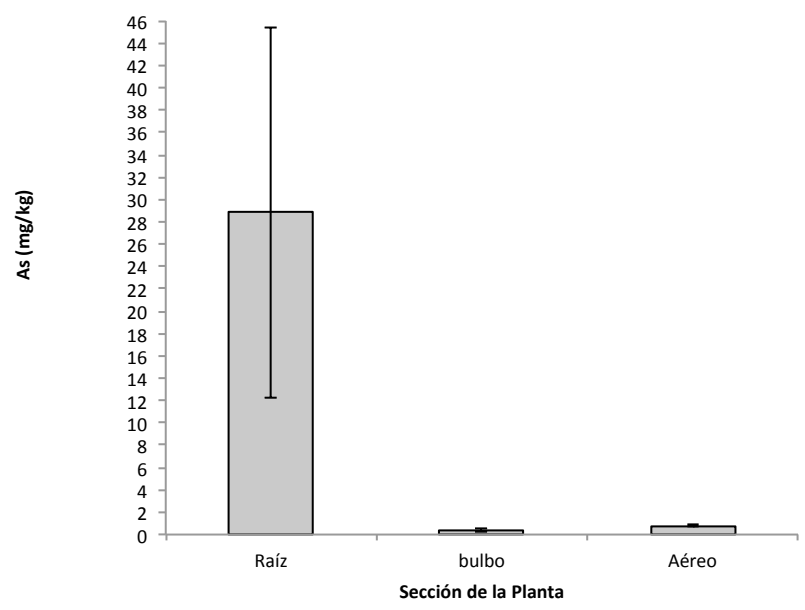

Fig. 7. Arsénico total $(\mathrm{mg} / \mathrm{kg})$ en plantas de cebolla

El Codex Alimentarius ha establecido para el arroz el límite máximo permisible de $0.2 \mathrm{mg} / \mathrm{kg}$ de As-tot (FAO/OMS 2011). Asimismo, en 2015 el Codex Alimentarius estableció que las hortalizas presentan concentraciones promedio de As-tot en un rango de 0.008 a $0.61 \mathrm{mg} / \mathrm{kg}$. (FAO/OMS 2015). Si se considera el LMP establecido para arroz, la cebolla lo superó, no obstante, la concentración media de As-tot en bulbo en esta investigación quedó dentro del rango de concentración definido a nivel global en hortalizas en el Codex Alimentarius. En el caso del chile quedó tanto por debajo del LMP establecido para arroz $(0.2 \mathrm{mg} / \mathrm{kg})$, así como se encuentra dentro del rango global de As-tot en hortalizas $(0.008$ a $0.61 \mathrm{mg} / \mathrm{kg})$.

En general la capacidad del chile para almacenar As-tot fue en el orden de raíz $>$ tallo $>$ hoja $>$ fruto; en la cebolla el orden fue raíz $>$ bulbo $>$ tallo (Rosas-Castor et al. 2014). 
El As se presenta en mayor concentración en la raíz debido a que las diversas especies de As ingresan por medio de las proteínas de transporte en la membrana de la raíz. Las similitudes en la estructura química entre del arsenato y el fosfato, y entre del arsenito y el ácido silícico, rigen su entrada en las celdas de la raíz.(Punshon et al. 2017).

Es importante acentuar que la acumulación de As en las plantas puede estar afectada por muchos factores, incluyendo el tipo de planta, los métodos de aplicación y los tipos de fertilizantes, la concentración de As en el agua de riego, las prácticas de riego y la especie de As presente, entre otros (Bundschuh et al. 2012). La transferencia de As del suelo y el agua a la parte comestible de la planta es una clave en la ruta de entrada del As a la cadena alimenticia (Rosas-Castor et al. 2014).

\section{CONCLUSIONES}

El As es un elemento presente en el Distrito de Riego 005 en el centro sur del estado de Chihuahua. En la zona norte de esta región se encontró As-tot en agua en seis sitios a niveles que pueden ocasionar un riesgo para la producción agrícola (As en pozos de agua $>100 \mathrm{mg} / \mathrm{L}$ ). No se encontró variación estacional en la concentración de As en agua.

La presencia de As-tot en el suelo fue relevante, aunque en la mayoría de los sitios no se superaron los límites establecidos en México, se asoció la trazabilidad del metaloide del agua al suelo. El As, al estar presente en el agua y el suelo, fue ser absorbido por las plantas; aunque se concentró mayormente en la raíz de las plantas, el chile y la cebolla demostraron que tienen la capacidad de traslocar y almacenar As en la parte comestible.

Para definir el riesgo de introducción de este metaloide a la cadena trófica por ingesta de alimentos, resulta necesario identificar la especiación del metaloide tanto en el agua como en el suelo y en las plantas, así como la formación de compuestos en el suelo con $\mathrm{Fe}$ o $\mathrm{Al}$, que pueden ayudar a comprender mejor la trazabilidad del metaloide.

\section{AGRADECIMIENTOS}

Se agradece al Consejo Nacional de Ciencia y Tecnología de México (CONACyT) por el apoyo económico recibido para la realización de esta investigación proyecto CB-2014/240849 y convenio I010/532/2014. Asimismo, se agradece al Ing. Jesús
Carmona Prado egresado de la Facultad de Ciencias Químicas de la Universidad Autónoma de Chihuahua, por su compromiso para la realización de este proyecto.

\section{REFERENCIAS}

Armienta M.A., Segovia, N. (2008). Arsenic and fluoride in the groundwater of Mexico. Environ. Geochem. Health. 30, 345-353.

DOI: $10.1007 / \mathrm{s} 10653-008-9167-8$.

Arreguín-Cortés F.I., Chávez-Guillén R., Soto-Navarro P.R. (2013). Una revisión de la presencia de arsénico en el agua subterránea en México. Comisión Nacional del Agua/Secretaría de Medio Ambiente y Recursos Naturales, CONAGUA/SEMARNAT, 11 pp.

Bundschuh J., Nath B., Bhattacharya P., Liu C.W., Armienta M.A., Moreno López M.V., López D.L., Jean J.S., Cornejo L., Lauer Macedo L.F., Filho A.T. (2012). Arsenic in the human food chain: the Latin American perspective. Sci Total Environ. 429: 92-106. DOI:10.1016/j.scitotenv.2011.09.069

Caballero-Gutiérrez P.U., Carrillo-Rodríguez J.C., Gomez-Ugalde R.M., Jerez-Salas M.P. (2010). Presencia de arsénico en pozos y en cultivos en Oaxaca, México. Agronomía Mesoamericana 21(1):177-184. DOI:10.15517/am.v21i1.4923.

Carbonell Barrachina A.A., Burló Carbonel F.M., Mataix Beneyto J.J. (1995). Arsénico

en el sistema suelo-planta. Universidad de Alicante, Espagrafic, España, 139 pp.

Chandra Santra S., Chandra Samal A., Bhattacharya P., Banerjee S., Biswas A., Majumdar J. (2013). Arsenic in food chain and community health risk: a study in Gangetic West Bengal. Procedia Environ Sci. 18: 2-13. DOI:10.1016/j.proenv.2013.04.002

CONAGUA. (2016). Ley federal de derechos disposiciones aplicables en materia de aguas nacionales. Comisión Nacional del Agua, Diario de la Federación, México, 168 pp.

Dahal B.M., Fuerhacker M., Mentler A., Karki K.B., Shrestha R.R., Blum W.E.H. (2008). Arsenic contamination of soils and agricultural plants through irrigation water in Nepal. Environ Pollut. 155 (1): 157-163. DOI: 10.1016/j.envpol.2007.10.024

Espino-Valdés M.S., Barrera-Prieto Y., Herrera-Peraza E. (2009). Presencia de arsénico en la sección norte del acuífero Meoqui-Delicias de Estado de Chihuahua. Tecnociencia Chihuahua 3 (1): 8-18.

FAO/OMS. (2011). Documento de debate sobre el arsénico en el arroz. Organización de las Naciones Unidas para la Alimentación y la Agricultura/Organización Mundial 
de la Salud, Codex Alimentarius, Roma, Italia, 28 pp.

FAO/OMS. (2015). Norma general para los contaminantes y las toxinas presentes en alimentos. Organización de las Naciones Unidas para la Alimentación y la Agricultura/Organización Mundial de la Salud, Codex Alimentarius, $62 \mathrm{pp}$.

FAO/OMS. (2017). Programa conjunto FAO/OMS sobre normas alimentarias Comisión del Codex Alimantarius. Organización de las Naciones Unidas para la Alimentación y la Agricultura/Organización Mundial de la Salud, Codex Alimentarius, Río de Janeiro, Brasil, $66 \mathrm{pp}$.

Feng Q., Zhang Z., Chen Y., Liu L., Zhang Z., Chen C. (2013). Adsorption and desorption characteristics of arsenic on soils: kinetics, equilibrium, and effect of $\mathrm{Fe}$ $(\mathrm{OH})_{3}$ colloid, $\mathrm{H}_{2} \mathrm{SiO}_{3}$ colloid and phosphate. Procedia Environ Sci.18, 26-36.

DOI: 10.1016/j.proenv.2013.04.005.

González-Horta C., Ballinas-Casarrubias L., SánchezRamírez B., Ishida M., Barrera-Hernández A., Gutiérrez-Torres D., Zacarias O., Saunders R., Drobná Z., Mendez M., García-Vargas G., Loomis D., Stýblo M., Del Razo L.M.A. (2015). Concurrent exposure to arsenic and fluoride from drinking water in Chihuahua, México. Int J Environ Res Public Health. 12 (5): 45874601. DOI:10.3390/ijerph120504587

INEGI. (2007). Panorama agropecuario en Chihuahua. Censo Agropecuario 2007. Instituto Nacional de Estadística y Geografía, Chihuahua, México.

INEGI. (2013). Instituto Nacional de Estadística y Geografía. http://www.inegi.org.mx/ (acceso 29-Diciembre-2016).

Kabata-Pendias A. (2011). Trace Elements in Soils and Plants. Fourth edition. Boca Raton, CRC Press, New York, USA, 505 pp.

Litter M.I., Armienta M.A., Farías S.S. (2009). Metodologías analíticas para la determinación y especiación de arsénico en aguas y suelos. Iberoarsen, CYTED, Buenos Aires, Argentina, 241 pp.

Minitab 17. (2018). Descargas. http://www.minitab.com/ es-mx/downloads/.

Moreno J.A. (2008). Sistemas y análisis de la información geográfica. Manual de autoaprendizaje con ArcGis. Ra-Ma, Segunda Edición. Madrid, España, 940 pp.

Olmos-Márquez M.A. (2011). Remoción de arsénico en el agua por fitorremediación con Eleocharis macrostachya en humedales construidos de flujo subsuperficial. Chihuahua. Centro de Investigación en Materiales Avanzados S.C. (CIMAV), Chihuahua, México, $99 \mathrm{pp}$.

Ortega García J., Lugo Sepúlveda R.E., Espinoza Ojeda E. (2010). Análisis de arsénico en papa usando un arsenómetro portátil. Invurnus 5 (1): 28-33.
Prieto-García F., Callejas Hernández J., Lechuga M.A. (2005). Acumulación de tejidos vegetales de arsénico proveniente de aguas y suelos de Zimapán estado de Hidalgo, México. Bioagro. 17(3):129-135.

Punshon T., Jackson B.P., Meharg A.A., Warczack T., Scheckel K., Guerinot M.L. (2017). Understanding arsenic dynamics in agronomic systems to predict and prevent uptake by crop plants. Sci Total Environ. 581, 209-220.

DOI: 10.1016/j.scitotenv.2016.12.111.

Rosas-Castor J. M., Guzmán-Mar J. L., Alfaro-Barbosa J.M., Hernández-Ramírez A., Pérez-Maldonado I.N., Caballero-Quintero A., Hinojosa-Reyes L. (2014). Evaluation of the transfer of soil arsenic to maize crops in suburban areas of San Luis Potosi, Mexico. Sci. Total Environ, 497, 153-162.

DOI: 10.1016/j.scitotenv.2014.07.072.

SCFI. (2001). NMX-AA-051-SCFI. Análisis de agua medición de metales por absorción atómica en aguas naturales, potables, residuales y residuales tratadasmétodo de prueba. Secretaría de Comercio y Fomento Industrial. Diario Oficial de la Federación, México.

SCFI. (2006). NMX-AA132-SCFI. Muestreo de Suelos para la identificación y la cuantificación de metales y metaloides, y manejo de la muestra. Secretaría de Comercio y Fomento Industrial. Diario Oficial de la Federación, México.

SEMARNAT/SSA. (2004). NOM-147-SEMARNAT/ SSA1. Que establece criterios para determinar las concentraciones de remediación de suelos contaminados por arsénico, bario, berilio, cadmio, cromo hexavalente, mercurio, níquel, plata, plomo, selenio, talio y/o vanadio. Secretaría de Medio Ambiente y Recursos Naturales/ Secretaría de Salud, Diario Oficial de la Federación, México.

Shraim A.M. (2017). Rice is a potential dietary source of not only arsenic but also other toxic elements like lead and chromium. Arab. J. Chem. 10 (2), 3434-3443. DOI:10.1016/j.arabjc.2014.02.004

SSA. (1993). NOM-014-SSA1. Procedimientos sanitarios para el muestreo de agua para uso y consumo humano en sistemas de abastecimiento de agua públicos y privados. Secretaría de Salud. Diario Oficial de la Federación, México.

SSA. (1994). NOM-127-SSA1. Agua para uso y consumo humano. Límites permisibles de calidad y tratamientos a que debe someterse el agua para su potabilización. Secretaría de Salubridad y Asistencia. Diario Oficial de la Federación, México.

Suresh K. R., Nagesh, M. A. (2015). Experimental studies on effect of water and soil quality on crop yield. Aquatic Procedia, 4, 1235-1242.

DOI: $10.1016 /$ j.aqpro.2015.02.161 
Vega-Gleason S. (2013). Riesgo sanitario ambiental por la presencia de arsénico y fluoruros en los acuíferos de México. Comisión Nacional del Agua, CONAGUA, México, 15 pp.
Yousif A.M., Zaid O.F., Ibrahim I.A. (2016). Fast and selective adsorption of $\mathrm{As}(\mathrm{V})$ on prepared modified cellulose containing $\mathrm{Cu}(\mathrm{II})$ moieties. Arab. J. Chem. 9 (5): 607-615. DOI:10.1016/j.arabjc.2015.02.004. 